\title{
Ictal semiology of epileptic seizures with insulo-opercular genesis
}

\author{
Eva Martinez-Lizana ${ }^{1}\left[\right.$ D $\cdot$ Armin Brandt $^{1} \cdot$ Niels A. Foit ${ }^{2} \cdot$ Horst Urbach $^{3} \cdot$ Andreas Schulze-Bonhage $^{1}$
}

Received: 30 July 2021 / Revised: 15 October 2021 / Accepted: 15 November 2021 / Published online: 23 November 2021

(c) The Author(s) 2021

\begin{abstract}
Objective Epileptic seizures with insular genesis are often difficult to distinguish from those originating in the temporal lobe due to their complex and variable semiology. Here, we analyzed differentiating characteristics in the clinical spectrum of insulo-opercular seizures.

Methods Ictal semiology in patients with a diagnosis of insulo-opercular epilepsy (IOE) based on imaging of epileptogenic lesions or electrophysiological evidence of an insulo-opercular seizure origin was retrospectively analyzed and compared to age-matched controls with mesial temporal lobe epilepsy (MTE).

Results Forty-six IOE and 46 matched MTE patients were included. The most prominent ictal features in IOE were focal motor phenomena in $80.4 \%$ of these patients. Somatosensory sensations, version, tonic and clonic features, when present, were more frequent contralateral to the SOZ in MTE patients, while they occurred about equally often ipsilateral and contralateral to the SOZ in IOE patients. Ipsilateral manual automatisms were significantly more frequent in MTE patients than in IOE $(p=0.010)$. Multivariate analysis correctly identified IOE in $78.3 \%$ and MTE in $84.8 \%$ using five semiologic features (Chi-square $=53.79$ with 5 degrees of freedom, $p<0.0001$ ). A subanalysis comparing patients with purely insular lesions with MTE patients using only the earliest ictal signs showed that somatosensory sensations are significantly more frequent in insular epilepsy $(p=0.010)$, while automatisms were significantly more frequent in MTE patients $(p=0.06)$.

Significance Our study represents the first in-depth analysis of ictal semiology in IOE compared to MTE. Use of these differentiating characteristics can serve for a correct syndrome classification and to steer appropriate diagnostic and local therapeutic procedures.
\end{abstract}

Keywords Insulo-opercular epilepsy $\cdot$ Semiology $\cdot$ Epilepsy surgery $\cdot$ Video-EEG $\cdot$ Insular cortex

\section{Introduction}

Among focal epilepsies, insulo-opercular epilepsy (IOE) poses particular problems in its identification and treatment. The insular cortex is integral part of multiple functional systems, ranging from sensory processing to autonomic and motor control, with widespread connectivity [1, 2]. Epileptic activity may thus result in multifaceted clinical appearance of seizures, rendering a differentiation from seizures of

Eva Martinez-Lizana

eva.martinez@uniklinik-freiburg.de

1 Epilepsy Center, Medical Center, University of Freiburg, Breisacher Str. 64, 79106 Freiburg im Breisgau, Germany

2 Department of Neurosurgery, Medical Center, University of Freiburg, Freiburg im Breisgau, Germany

3 Department of Neuroradiology, Medical Center, University of Freiburg, Freiburg im Breisgau, Germany temporal origin a problem $[3,4]$. The electrophysiological access to insular discharges is severely hampered: covered by the opercula, scalp EEG does not provide direct recordings from the insular cortex, and only activity propagated to the dorsolateral convexity can be seen. Even seizures may lack a well localizable EEG correlate, which has led to confusion with non-epileptic, psychogenic seizures [5, 6]. Intracranial recordings need the use of depth electrodes; their implantation does carry an increased risk due to the multiple branches of the middle cerebral artery. Thus, most studies using SEEG are limited by severe undersampling of the insular cortex with an average of only 1-18 electrode contacts in the complete lobe in publications from Afif [7], Isnard [8, 9] and Blauwblomme [10]. In recent studies increased coverage of the insular cortex has been achieved, but the identification of potentially epileptogenic lesions on MR imaging often still constitutes the best evidence for seizure generation in the insular cortex; like in 
other localizations, however, even best available imaging techniques do not reveal a lesion in all patients $[11,12]$.

An improved understanding of semiological features of insulo-opercular epilepsy thus remains essential to raise suspicion for this syndrome. Unfortunately, the patient numbers included in previous reports on insulo-opercular seizure semiology have frequently been low [13-15]. According to previous reports, ictal semiology of insulo-opercular seizures comprises various non-specific patterns including somatosensory/viscerosensory, autonomic, speech-related abnormalities, fear and a variety of motor signs including automatisms [16, 17]. Importantly, these semiological features are not exclusive to IOE but are also found in frontal and temporal lobe epilepsy.

To date, no study has compared IOE to other common forms of focal epilepsy, although there is a clear need to improve identification of potential epilepsy surgery candidates or identify appropriate targets for focal stimulation. The differentiation of insulo-opercular seizures from TLE represents the most frequently encountered challenge, as an important fraction of patients who failed temporal lobe surgery may have been misdiagnosed with IOE or feature an extension of the epileptogenic zone into the insula [13, 18].

A better understanding of the neuroanatomical basis of insular ictal semiology will therefore likely improve candidate selection for resective procedures or electrode placement $[3,5,19]$. The purpose of this study is to assess seizure semiology of IOE more precisely and to identify factors/patterns segregating IOE from mesiotemporal epilepsy (MTE).

\section{Methods}

Forty-six patients with a diagnosis of IOE at the Freiburg Epilepsy Center between 2003 and 2019 were retrospectively identified from medical records. The inclusion of patients in the IOE group was based on either MR evidence of an insular lesion which was regarded as typically epileptogenic (e.g., cortical dysplasia, tumors, cavernomas) and concordant information from scalp-EEG (fronto-temporal ictal patterns) or if there was stereo-electroencephalography (SEEG) evidence for insulo-opercular seizure generation (in two non-lesional cases and in additional ten patients with multiple lesions or lesions extending beyond the insular border to ascertain insulo-opercular seizure onset). A part of the epileptogenic lesions extended beyond the strict limits of the insular cortex in the adjacent opercula.

This IOE cohort was compared to 46 age-matched patients with previous successful selective amygdalohippocampectomy for drug-refractory mesial temporal lobe epilepsy (MTE) (Engel I outcome at a minimum of 12 months after surgery). Temporal lobe epilepsy was selected as control group as insulo-opercular seizure origin has been found to be a relevant cause of outcome failures of temporal resection [4].

Standardized clinical evaluation included detailed history and physical examination, MRI and long-term video-EEG monitoring (VEEG). Additionally, SEEG provided evidence of insular origin in 12 IOE patients ( 2 non-lesional cases; 1 patient with left hippocampal sclerosis and focal cortical dysplasia involving left insular and adjacent frontal and temporal cortex; 3 patients with an FCD involving insular and adjacent frontal and temporal regions; 1 patient with an extensive FCD involving insular and adjacent temporal and parietal regions; 1 patient with multiple dysplastic regions of left insula, right angular gyrus and right frontal cortex, 1 patient with tuberos sclerosis and tubers left fronto-insular, in cingulate cortex anterior, in frontal superior and medius gyrus; 1 patient with a cavernous hemangioma in the right putamen and a right temporo-insular ganglioglioma WHO grad I; 1 patient with a purely left insular FCD yet more extended ictal EEG patterns, findings on FDG-PET and ictal SPECT, and 1 patient with fronto-insular FCD who did not achieve seizure-freedom after a first surgery). Seven of the MTE patients with electroclinical discordance had undergone SEEG. MRI was performed with $1.5 \mathrm{~T}$ (Siemens Magnetom Vision) or $3 \mathrm{~T}$ scanners (Siemens Magnetom Trio or Prisma). T1-weighted sequences with and without gadolinium-diethylenetriaminepentaacetic acid (DTPA), T2w, fluid-attenuated inversion recovery (FLAIR) and magnetization-prepared rapid gradient echo (MPRAGE) sequences were obtained. To assess mesiotemporal lobe integrity, $\mathrm{T} 2 \mathrm{w}$ axial and coronal images with a modified angulation parallel to the long axis of the hippocampus were acquired. Patients initially underwent VEEG at the Epilepsy Centers of Heidelberg, Kehl-Kork or Freiburg and were later referred to the University Medical Center Freiburg for presurgical evaluation and intracranial recordings, if necessary SEEG implantation was guided by non-invasive EEG data and covered the hypothetical epileptogenic zone(s) as well as the lesional area, if identified. All data were retrospectively assessed by review of medical records and video-EEG recordings.

Semiological features were analyzed at the patient level and classified in accordance with the latest operational classification of seizure types by the International League Against Epilepsy [20] into: somatosensory (including perioral and throat sensations), olfactory, auditory, gustatory, cephalic or epigastric sensation, déjà-vu, fear, autonomic alterations (including feeling of warmth, flush, ictal tachycardia or bradycardia, hypersalivation and nausea), aphasia, ictal speech, hyperkinetic or focal motor activity (including versive, clonic or tonic posturing), automatisms (oral or manual), behavioral arrest, and postictal aphasia. Focal seizures progressing to bilateral tonic-clonic seizures were also documented. Somatosensory sensations, manual automatisms, versive, clonic, and tonic activity were additionally classified into not present, present 
but non-lateralizing (bilateral or alternating between right and left), ipsi- or contralateral to the seizure onset zone (SOZ). Semiological features were considered initial if they were the earliest feature in at least one seizure of a given patient. In the case that different seizures had more than three different initial semiological signs, the three features most frequently occurring at the start of seizures were used for analysis.

A combined analysis was performed using lateralizing features expected to be contralateral to the SOZ (somatosensory sensations, versive, clonic, and tonic movements). The score was considered ipsilateral if at least one element was lateralized ipsilateral and none contralateral; contralateral, if at least one element was lateralized contralateral and none ipsilateral; and non-lateralizing, if at least one ipsilateral and one contralateral involvement was present, respectively.

Data analysis was performed using R version 4.0.2 [21]. Frequencies of semiological features were compared between IOE and MTE patients using Fisher's exact test (2-tailed). Features showing significantly different frequencies $(p<0.05)$ were included in an initial logistic regression model. As the data showed quasi-complete separation [22], a bias-reduced general linear model (R-package 'brglm2', version 0.6.2 [23]) was used for this purpose. Variables without a significant independent contribution to the initial multivariate model were then excluded by backward elimination.

To systematically identify co-occurring features, we performed a hierarchical agglomerative cluster analysis using complete linkage [24]. The distance between pairs of features was computed as the number of patients who exhibited different behavioral patterns for these features (one present and one absent) divided by the total number of patients ( 46 in each group).

To further investigate lesion-semiology associations, an expert rater (NAF) manually segmented structural lesions on individual volumetric T1w MRI in native space with ITK-snap for patients in which volumetric imaging data were available $(n=30)$ [25]. Following structural pre-processing of individual T1w images including segmentation using SPM12 (fil. ion.ucl.ac.uk/spm) default routines, binary lesion maps were normalized to Montreal Neurological Institute (MNI) stereotaxic space with the transformation matrices obtained during preprocessing. Group-specific lesion maps were created with MRIcroGL [26] (Fig. 1a).

The study was approved by the Ethics Review Board of the University Medical Center Freiburg and the patients gave their written consent that the data collected for presurgical epilepsy diagnosis may be used for scientific evaluation.

\section{Results}

This series consists of 46 patients with IOE ( 27 women, mean age at evaluation 28 years; mean age at onset 16 years, mean duration of epilepsy 12 years) and 46 MTE patients with a lesion in the same hemisphere and approximately (mean 31 years) the same age at presurgical workup. Further demographic and clinical data are summarized in Table 1. Based on the electroclinical and imaging data, seizures originated in the right hemisphere in 26 patients and in the left hemisphere in 20 patients.

In IOE patients, focal motor phenomena were the most frequent ictal semiological manifestation (in $80.4 \%$ of patients), including tonic posturing in $63.0 \%$, clonic movements in $58.7 \%$, version in $23.9 \%$ of patients, and hyperkinetic features in $21.7 \%$. Behavioral arrest occurred in $54.3 \%$ of the patients, oral automatisms in $43.5 \%$ and manual automatisms in $39.1 \%$. Autonomic signs and symptoms were reported in $58.7 \%$ of the patients, including feeling of warmth in $13.0 \%$, flush in $19.6 \%$, ictal tachycardia/ bradycardia in $30.4 \%$ and nausea in $8.7 \%$. Somatosensory focal aware seizures were recorded in $32.6 \%$, olfactory or gustatory sensations in $17.4 \%$, auditory sensations in $8.7 \%$, déjà-vu in $8.7 \%$, fear in $10.9 \%$, cephalic in $21.7 \%$ and epigastric sensations in $19.6 \%$ of the patients. Ictal or postictal aphasia was present in $32.6 \%$ and ictal speech in $4.3 \%$ of the patients.

When comparing with MTE patients, somatosensory symptoms were significantly more frequent in patients with IOE (32.6\% vs. $10.9 \%, p=0.021)$, as well as focal motor phenomena $(80.4 \%$ vs. $52.2 \%, p=0.008)$ and hyperkinetic features $(21.7 \%$ vs. $0 \%, p=0.001)$. Auditory sensations were found only in IOE, not in MTE, whereas olfactory or gustatory sensations were found in both IOE (17.4\%) and MTE (6.5\%). In contrast, epigastric sensations were more frequent in MTE patients (19.6\% vs. $50.0 \%, p=0.004)$, as were ictal automatisms $(54.3 \%$ vs. $89.1 \%, p<0.001)$, behavioral arrest $(54.3 \%$ vs. $84.8 \%, p=0.003)$ and ictal speech $(4.3 \%$ vs. $26.1 \%, p=0.007)$. Autonomic features were observed in a high percentage of both groups. Particularly, feeling of warmth was found in 2 patients with MTE and 6 patients with IOE, flush in 6 patients with MTE and 9 patients with IOE, ictal tachycardia or bradycardia in 17 patients with MTE and 14 patients with IOE, hypersalivation in 1 patient with MTE and 5 patients in IOE and nausea in 6 patients with MTE and 4 patients with IOE. Evolution to bilateral tonic-clonic seizures tended to appear more frequently in patients with MTE (67.4\%) than in patients with IOE $(52.2 \%)$. Results of univariate analyses of the semiologic features of IOE and MTE patients are shown in Table 2.

Patients with purely insular lesions $(n=15)$ were separately analyzed and compared with the MTE patients 
Fig. 1 Examples of patients included based on a visible epileptogenic lesion: a, b Cavernoma in the left insula. Axial T2-MRI imagines of the lesion prior to epilepsy surgery (a) and following lesionectomy (b). c, $\mathbf{d}$ Focal cortical dysplasia in the left insula. Coronal T2-MRI imagines of the lesion prior to epilepsy surgery (c) and following lesionectomy (d); $\mathbf{e , ~} \mathbf{f}$ Angiocentric glioma WHO I in the left anterior insula. Coronal T2-MRI imagines of the lesion prior to epilepsy surgery (e) and following lesionectomy (f). g From the 44 patients with a MR-based evidence for insular seizure origin, we performed heat map of lesion extension in patients with 3D MRI datasets available $(n=30)$. Individual binary lesion maps are superimposed onto the MNI152 - template. Spectral colors demonstrate degrees of structural lesion overlap from violet $=1$ patient to red $=$ all patients in the cohort. Note that in some patients, the lesion extended into the adjacent operculum
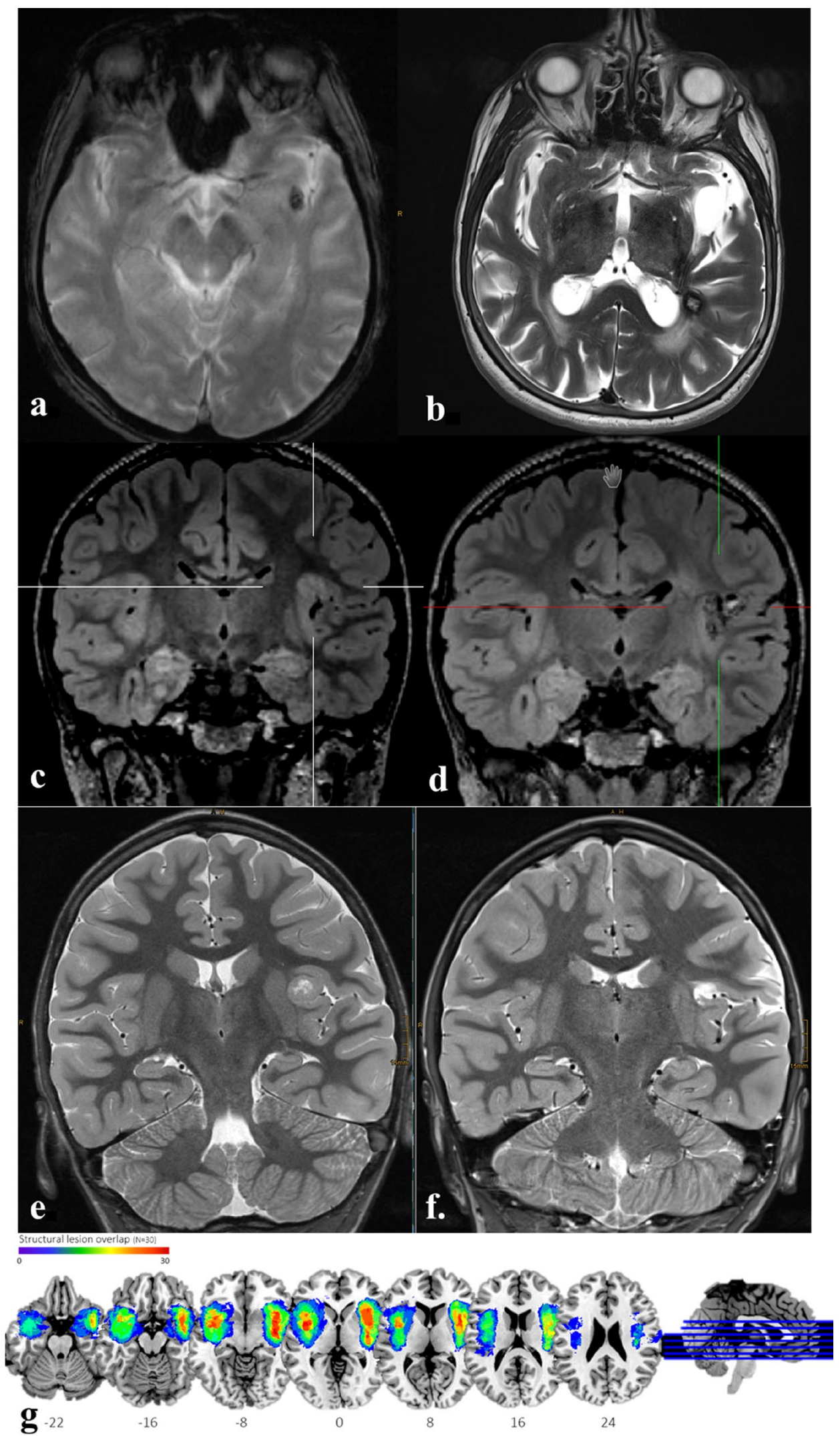
Table 1 Demographics and clinical findings in patients with insuloopercular epilepsy and the matched-pair control patients with mesiotemporal epilepsy

\begin{tabular}{lll}
\hline Characteristics & $\begin{array}{l}\text { Insulo-opercular epi- } \\
\text { lepsy patients }(n=46)\end{array}$ & $\begin{array}{l}\text { Mesiotemporal } \\
\text { epilepsy patients } \\
(n=46)\end{array}$ \\
\hline $\begin{array}{ll}\text { Sex (male/female) } \\
\text { Age at epilepsy onset } \\
\quad \text { years) mean/median }\end{array}$ & $19 / 27$ & $19 / 27$ \\
Duration of epilepsy, & & $13.5 / 10.0$ \\
years & & \\
Mean & 12 & \\
Range & $0-40$ & 18 \\
Lesion on MRI, $n(\%)$ & $44(95.7)$ & $0-43.4$ \\
Cavernoma & 2 & $46(100)$ \\
DNET & 2 & 1 \\
FCD & 21 & 0 \\
Gliosis & 1 & 3 \\
Infarction & 1 & 0 \\
Hippocampal Sclerosis & 0 & 0 \\
Tuber & 2 & 36 \\
Other tumors & 17 & 0 \\
\hline
\end{tabular}

(Supplementary Table 1). Similarly to the previous analysis, somatosensory symptoms were significantly more frequent in patients with insular epilepsy (IE) $(47.6 \%$ vs. $10.9 \%, p=0.006)$. Focal motor phenomena ( $80 \%$ vs. $52.2 \%$ ) remained more frequent in patients with IE than in MTE, but the difference was no longer significant. Still in the subgroup with 15 patient with IE there were auditory sensations and hyperkinetic features but not in MTE, whereas olfactory or gustatory sensations occurred in both IOE (20\%) and MTE (6.5\%). Ictal automatisms were more frequent in MTE patients than in IE patients $(89.1 \%$ vs. $40 \%$, as were instances of behavioral arrest ( 84.8 vs. $33.3 \% p<0.001)$. Ictal speech was present only in MTE (26.1\%). Epigastric sensations tended to be more frequent in MTE patients than in IE patients (50\% vs. 26.7\%) but this difference was not significant.

When taking into account only the earliest (up to three) ictal signs of patients with purely insular lesions and patients with MTE, respectively, somatosensory symptoms were significantly more frequent in patients with IOE than in patients with MTE. In contrast, Automatisms were more frequent in MTE patients. Initial focal motor phenomena appeared in $47 \%$ of patients with purely insular lesions and in $22 \%$ of patients with MTE (Supplementary Table 2).

Results of the univariate analysis of lateralizing SF in IOE and MTE patients are shown in Table 3. Ipsilateral manual automatisms were significantly more frequent in MTE patients than in IOE $(p=0.010)$. We performed a combined univariate analysis with somatosensory sensations, version, tonic and clonic features (see "Methods"). If one or more of the features were present, they were predominantly present contralateral to the SOZ in MTE patients whereas in IOE patients, they occurred about equally often ipsilateral as contralateral to the $\mathrm{SOZ}$ ( $p=0.0217$, Table 3$)$.

Results of a logistic regression analysis including semiologic features with a $p$-value $<0.05$ in the univariate analysis is shown in Table 4 . The $\chi^{2}$ for the overall likelihood ratio test of the model was $64.1(p<0.0001)$. Variables with an
Table 2 Semiologic features during seizures of patients with insulo-opercular epilepsy or mesiotemporal epilepsy

\begin{tabular}{lccc}
\hline Semiologic feature & $\begin{array}{l}\text { Insulo-opercular epilepsy, } n \\
(\%) n=46\end{array}$ & $\begin{array}{l}\text { Mesiotemporal } \\
\text { epilepsy, } \\
n(\%) n=46\end{array}$ & $\begin{array}{l}p \text { value (two-tailed } \\
\text { Fisher's exact test) }\end{array}$ \\
\hline Somatosensory & $15(32.6)$ & $5(10.9)$ & 0.021 \\
Olfactory or gustatory & $8(17.4)$ & $3(6.5)$ & 0.117 \\
Auditory & $4(8.7)$ & $0(0)$ & 0.197 \\
Déjà-vu & $4(8.7)$ & $0(0)$ & 0.117 \\
Fear & $5(10.9)$ & $4(8.7)$ & 1.000 \\
Cephalic & $10(21.7)$ & $7(15.2)$ & 0.592 \\
Epigastric & $9(19.6)$ & $23(50.0)$ & 0.004 \\
Autonomic & $27(58.7)$ & $35(76.1)$ & 0.119 \\
Ictal or postictal aphasia & $15(32.6)$ & $25(54.3)$ & 0.058 \\
Ictal speech & $2(4.3)$ & $12(26.1)$ & 0.007 \\
Automatisms & $25(54.3)$ & $41(89.1)$ & $<0.001$ \\
Hyperkinetic & $10(21.7)$ & $0(0)$ & 0.001 \\
Focal motor & $37(80.4)$ & $24(52.2)$ & 0.008 \\
Behavioral arrest & $25(54.3)$ & $39(84.8)$ & 0.003 \\
Evolution to BTCS* & $24(52.2)$ & $31(67.4)$ & 0.202 \\
\hline
\end{tabular}

*BTCS bilateral tonic-clonic seizure 
Table 3 Lateralizable semiologic features during seizures of patients with insulo-opercular epilepsy or mesiotemporal epilepsy

\begin{tabular}{|c|c|c|c|}
\hline Lateralizing semiologic feature & & $\begin{array}{l}\text { Insulo-opercular epi- } \\
\text { lepsy, } n(\%) n=46\end{array}$ & $\begin{array}{l}\text { Mesiotemporal } \\
\text { epilepsy, } n(\%) \\
n=46\end{array}$ \\
\hline \multirow[t]{4}{*}{ Somatosensory } & None & $32(67.4)$ & $42(89.1)$ \\
\hline & Non-lateralizable & $10(21.7)$ & $4(8.7)$ \\
\hline & Ipsilateral & $3(6.5)$ & $1(2.2)$ \\
\hline & Contralateral & $2(4.3)$ & $0(0)$ \\
\hline \multirow[t]{4}{*}{ Manual automatisms } & None & $28(60.9)$ & $13(28.3)$ \\
\hline & Non-lateralizable & $5(10.9)$ & $3(6.5)$ \\
\hline & Ipsilateral & $7(15.2)$ & 19 (41.3) \\
\hline & Contralateral & $6(13.0)$ & $11(23.9)$ \\
\hline \multirow[t]{4}{*}{ Version } & None & $35(76.1)$ & $32(69.6)$ \\
\hline & Non-lateralizable & $3(6.5)$ & $2(4.3)$ \\
\hline & Ipsilateral & $3(6.5)$ & $1(2.2)$ \\
\hline & Contralateral & $5(10.9)$ & $11(23.9)$ \\
\hline \multirow[t]{4}{*}{ Clonic } & None & $26(56.5)$ & $29(63.0)$ \\
\hline & Non-lateralizable & $7(15.2)$ & $9(19.6)$ \\
\hline & Ipsilateral & $6(13.0)$ & $2(4.3)$ \\
\hline & Contralateral & $7(15.2)$ & $6(13.0)$ \\
\hline \multirow[t]{4}{*}{ Tonic } & None & $17(37.0)$ & $22(47.8)$ \\
\hline & Non-lateralizable & $11(23.9)$ & $8(17.4)$ \\
\hline & Ipsilateral & $9(19.6)$ & $1(2.2)$ \\
\hline & Contralateral & $9(19.6)$ & $15(32.6)$ \\
\hline \multirow{4}{*}{$\begin{array}{l}\text { Combined analysis with Somatosen- } \\
\text { sory, version, tonic and clonic }\end{array}$} & None & $6(13.0)$ & $16(34.8)$ \\
\hline & Non-lateralizable & $19(41.3)$ & $8(17.4)$ \\
\hline & Ipsilateral & $10(21.7)$ & $3(6.5)$ \\
\hline & Contralateral & $11(23.9)$ & $19(41.3)$ \\
\hline
\end{tabular}

Ipsilateral manual automatisms are significantly more frequent in patients with mesiotemporal epilepsy than in patients with insulo-opercular epilepsy $(p=0.010)$. The combined analysis with somatosensory, version, tonic and clonic symptoms showed that if one or more of the features were present, were predominantly present contralateral to the lesion in mesiotemporal patients whereas in insular patients, they occurred about equally often ipsilateral as contralateral to the lesion

Table 4 Multivariate logistic regression analysis of semiologic features that best differentiated between patients with insulo-opercular epilepsy and patients with mesiotemporal epilepsy

\begin{tabular}{lccc}
\hline Semiologic feature & Odds ratio & 95\% Confidence interval of odds ratio & $p$ value \\
\hline Epigastric & 0.303 & $0.094-0.980$ & 0.046 \\
Aphasia & 0.313 & $0.104-0.941$ & 0.039 \\
Ictal speech* & 0.021 & $0.001-0.433$ & 0.012 \\
Automotor & 0.144 & $0.037-0.562$ & 0.005 \\
Hyperkinetic* & 275.941 & $3.231-23,567.469$ & 0.013 \\
\hline Patients & Correct & Incorrect & No \\
\cline { 2 - 4 } & No & 78.3 & 10 \\
\hline Insulo-opercular epilepsy & 36 & 84.8 & 7 \\
Mesiotemporal epilepsy & 39 & 21.7 \\
\hline
\end{tabular}

Variables with an odds ratio greater than 1 are independently predictive for an IE. Variables with an odds ratio less than 1 are independently predictive for a MTE. Using this model with the five significant features $78.3 \%$ of insular patients and $84.8 \%$ of mesiotemporal patients were correctly identified

*Estimation of the odds ratio for the features 'Hyperkinetic' and 'Ictal speech' is unreliable, due to quasi-complete separation of the data set [41] 
odds ratio greater than 1 are independently predictive for IOE (hyperkinetic features). On the other hand, variables with an odds ratio less than 1 are independently predictive for MTE (epigastric sensations, aphasia, ictal speech and automotor features). Table 4 shows the reclassification accuracy when using the final multivariate logistic regression model. Using this model containing five features with significant contributions (epigastric sensations, aphasia, ictal speech, automotor and hyperkinetic or motor features), $78.3 \%$ of IOE patients and $84.8 \%$ of MTE patients were correctly identified.

Cluster analysis results are shown in Fig. 2 as dendrograms. While we could identify two separate clusters of symptoms in both IOE and MTE patients, feature associations differed between the two groups. In patients with IOE, cluster 1 was characterized by déjà-vu, auditory sensations, fear, ictal speech, cephalic and epigastric sensations, olfactory or gustatory sensations, somatosensory symptoms and hyperkinetic features. Cluster 2 was comprised of behavioral arrest, aphasia, automatisms, autonomic and focal motor features. In patients with MTE, the first cluster was characterized by déjà-vu, hyperkinetic features, olfactory, gustatory or auditory sensations, fear, somatosensory symptoms, cephalic sensations and ictal speech, while the second cluster was characterized by three sub-clusters consisting of behavioral arrest and automatisms, autonomic symptoms and epigastric sensations, and focal motor features and aphasia, respectively.

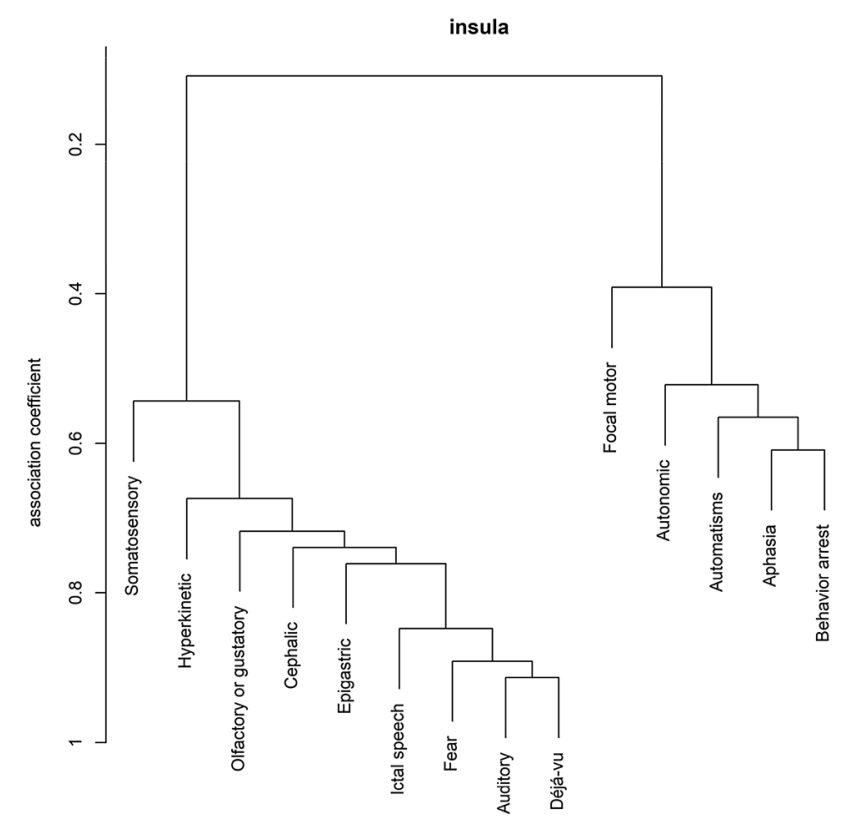

\section{Discussion}

We here analyzed the semiology of a large cohort of patients with insulo-opercular seizure generation. Evidence for the insula was based on an insular lesion in 34/46 cases, on an insular lesion and additional SEEG confirmation in 10/46 cases, and on SEEG in two non-lesional cases. As mentioned above, SEEG is limited in providing evidence of an insular origin due to an undersampling of the insular cortex with standard lateral approaches; it was thus implemented only in multilesional cases (e.g., tuberous sclerosis), when the limits of the lesion were not clear (e.g., some FCD) or in non-lesional cases.

Interestingly, the results of our study found seizure characteristics, which highly significantly allowed to separate the insulo-opercular seizures from mesiotemporal seizures. This supports the localizing role of semiologic features in presurgical evaluation [27-31]. In particular, the data may be helpful in pointing towards seizure generation beyond the temporal lobe.

Corroborating previous work, our study clearly demonstrates that insulo-opercular seizure semiology is very heterogeneous [31, 32]. Presence of focal motor phenomena was the most frequent semiological feature in agreement with one previous study [33], suggesting early spread from the insula to the primary motor cortex, premotor cortex and the frontal pole. This finding is in concordance with a systematic review on published cases of insular seizures

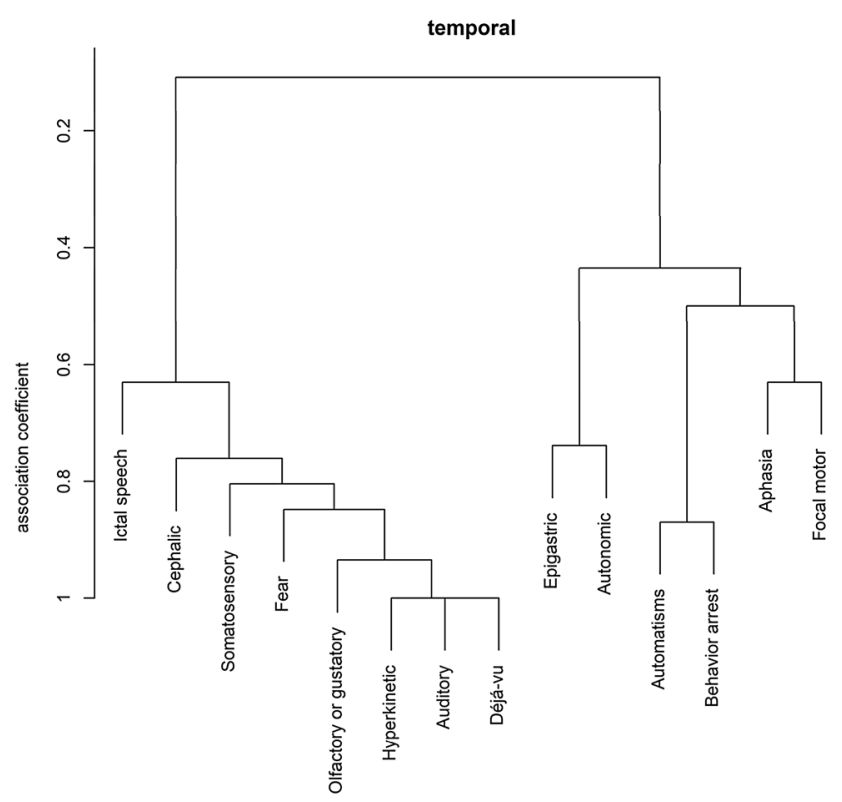

Fig. 2 Results of the cluster analysis. The left dendrogram shows features of the patients with insulo-opercular epilepsy and on the right features of the patients with mesiotemporal epilepsy 
[34]. The second most frequent seizure manifestation was somatosensory (including perioral and throat sensations) and autonomic symptoms (including bradycardia) [16, 17, 34]. Progression to bilateral tonic-clonic seizures was less frequent in other series than in our cohort, which may be partially related to tapering of antiepileptic drugs during video-EEG recordings in our patient sample.

Somatosensory symptoms were present significantly more frequently in IOE than MTE. Focal motor phenomena were only found significantly more frequently in IOE than MTE when analyzing all semiological signs. In the analysis of the earliest ictal signs, the difference was no longer significant. Our data support the hypothesis that in insular epilepsy, focal motor features more likely reflect activation of fronto-insular networks rather than being primarily insular seizure manifestations, in agreement with the studies of Singh and Isnard [2, 3].

In addition, we found auditory sensations in $8.7 \%$ of IOE; this is of interest and reflects the involvement of insula in auditory processing [35]. Of note, auditory symptoms were not present in the temporal cohort reflecting the strictly mesiotemporal seizure origin; this differentiation cannot be extended to temporal neocortical epilepsy which may involve the auditory cortex and belt. An interesting point is that IOE seizures had a lower lateralizing features compared to temporal lobe seizures. The lower lateralizing value of the ictal features in patients with IOE may be explained through the wide connectivity of the insula, including contralateral brain regions [36], possibly reflecting early contralateral propagation of the ictal activity [32] and bilateral representation within the insular cortex [37].

Hyperkinetic phenomena was the strongest predictive features for patients with IOE in the multivariate analysis. We observed hyperkinetic features in IOE in $21.7 \%$ of patients, which is similar as reported by Peltola et al. and Wang et al. $[16,17]$. This was the most distinguishing feature from a mesiotemporal onset. Of note, also temporal neocortical seizures arising from the temporal pole may present with hyperkinetic motor phenomena [38]. While we also found autonomic symptoms in a high proportion of patients with IOE (58.7\%) (see also [16]); their presence, however, did not separate IOE from MTE in which autonomic symptoms and signs were found in $76.1 \%$. Moreover, epigastric sensations and automatisms were significantly more frequent in MTE compared to IOE; and epigastric sensations, automatisms, ictal speech and aphasia were independently predictive of MTE in the multivariate analysis. In a model encompassing the features hyperkinetic, epigastric, aphasia, ictal speech and automatism, $78.3 \%$ of IOE patients and $84.8 \%$ of MTE patients were identified correctly. Previous studies with intracranial electrodes showed that there are distinct semiological subgroups in the insula [16, 31, 32]. This study did not separate insular subregions but joined semiological features from all IOE cases for differentiation from mesiotemporal onset. This reflects a variable extension of lesions within the insular cortex and connectivity within the insula and provides clinically relevant information for presurgical localization and planning of surgery.

The performed cluster analysis revealed that in both patient groups, subjective feelings (including somatosensory sensations but also fear, olfactory, gustatory, auditory, déjà-vu or cephalic sensations) and hyperkinetic features were associated in one cluster, behavioral arrest, aphasia, automatisms, autonomic features, and focal motor features in a second one. Ictal speech characterizes seizures generated in the non-dominant hemisphere, which may contribute to its positive association with reported subjective feelings in both patient groups, as awareness is more frequently preserved and subjective feelings thus experienced during and remembered after the seizure. We did not find obvious relationship between the semiological subclusters and anatomic subregions of the insula. This may reflect epileptogenic lesions extending not limited to a subarea of the insula but rather across the whole insula as well as a role of ictal propagation in the clinical manifestation of the seizures.

The insula is by no means an isolated functional center, as the term "insula" may suggest, rather the wide clinical spectrum of insular seizures reflects that the insula acts as a multimodal hub region with extensive structural and functional connections to other brain regions [36]. On the other hand, the anatomical organization of the insula per se is highly heterogeneous and its disruption by seizures may similarly elicit a wide spectrum of symptoms [2, 7]. While epigastric, olfactory, gustatory and auditory sensations may relate to a primary generation within the insular cortex [39, 40], behavioral arrest and automatisms likely reflect seizure spread through extensive connections to the temporal lobe; emotional and cognitive auras to the limbic regions; tonic, clonic or hyperkinetic phenomena and speech dysfunction to frontal regions [1].

The semiological features reported here, as well as the stereotypy of events, imaging and the knowledge that at least focal aware seizures may not be accompanied by scalp EEG correlates will also contribute to a better clinical judgement and differentiation between PNES and seizures of insular origin.

This study has several limitations. Some patients had a lesion which extended beyond the strict borders of the insula, particularly to one of the opercula. This reflects the clinical spectrum of patients presenting with insular seizures and was thus not chosen as an exclusion criterion. An extension of the SOZ beyond the peri-insular circular sulcus may favor the occurrence of motor seizures. Future prospective multicenter assessments in larger groups of patients may provide further insight of the role of adjacent regions for the newly identified semiological clusters, including the 
temporal sequence of symptoms, and the distinction of IOE to other regions of focal epileptogenesis.

Supplementary Information The online version contains supplementary material available at https://doi.org/10.1007/s00415-021-10911-0.

Acknowledgements The authors thank the patients and their caregivers.

Funding Open Access funding enabled and organized by Projekt DEAL. Salary support to NAF was provided by the German Research Foundation (DFG, FO996/1-1 to NAF) and the International League against Epilepsy outside the submitted work; ASB reports grants from European Union, grants from NIH, Grants from BMBF, Grants from Bial, Grants and personal fees from Precisis, personal fees from Arvelle, personal fees from Bial, personal fees from Eisai, personal fees from UCB, Grants from DFG, outside the submitted work; EML reports personal fees from Sociedad Andaluza de Epilepsia, outside the submitted work.

\section{Declarations}

Conflicts of interest Armin Brandt, Niels Alexander Foit, Andreas Schulze-Bonhage, Horst Urbach and Eva Martinez-Lizana report no disclosure relevant to this manuscript.

Ethical statement This study has been approved by the appropriate ethics committee and have therefore been performed in accordance with the ethical standards laid down in the 1964 Declaration of Helsinki and its later amendments.

Open Access This article is licensed under a Creative Commons Attribution 4.0 International License, which permits use, sharing, adaptation, distribution and reproduction in any medium or format, as long as you give appropriate credit to the original author(s) and the source, provide a link to the Creative Commons licence, and indicate if changes were made. The images or other third party material in this article are included in the article's Creative Commons licence, unless indicated otherwise in a credit line to the material. If material is not included in the article's Creative Commons licence and your intended use is not permitted by statutory regulation or exceeds the permitted use, you will need to obtain permission directly from the copyright holder. To view a copy of this licence, visit http://creativecommons.org/licenses/by/4.0/.

\section{References}

1. Ghaziri J, Tucholka A, Girard G, Houde JC, Boucher O, Gilbert $\mathrm{G}$ et al (2017) The corticocortical structural connectivity of the human insula. Cereb Cortex 27(2):1216-1228

2. Kurth F, Zilles K, Fox PT, Laird AR, Eickhoff SB (2010) A link between the systems: functional differentiation and integration within the human insula revealed by meta-analysis. Brain Struct Funct 214(5-6):519-534

3. Obaid S, Zerouali Y, Nguyen DK (2017) Insular Epilepsy: semiology and noninvasive investigations. J Clin Neurophysiol 34(4):315-323

4. Barba C, Minotti L, Job AS, Kahane P (2017) The insula in temporal plus epilepsy. J Clin Neurophysiol 34(4):324-327
5. Jobst BC, Gonzalez-Martinez J, Isnard J, Kahane P, Lacuey N, Lahtoo SD et al (2019) The insula and its epilepsies. Epilepsy Curr 19(1):11-21

6. Salado AL, Koessler L, DeMijolla G, Schmitt E, Vignal JP, Civit $T$ et al (2018) sEEG is a safe procedure for a comprehensive anatomic exploration of the insula: a retrospective study of 108 procedures representing 254 transopercular insular electrodes. Oper Neurosurg 14(1):1-8

7. Afif A, Minotti L, Kahane P, Hoffmann D (2010) Anatomofunctional organization of the insular cortex: a study using intracerebral electrical stimulation in epileptic patients. Epilepsia 51(11):2305-2315

8. Isnard J, Guenot M, Sindou M, Mauguiere F (2004) Clinical manifestations of insular lobe seizures: a stereo-electroencephalographic study. Epilepsia 45(9):1079-1090. https://doi.org/10. 1111/j.0013-9580.2004.68903.x

9. Isnard J, Guénot M, Ostrowsky K, Sindou M, Mauguière F (2000) The role of the insular cortex in temporal lobe epilepsy. Ann Neurol 48(4):614-623

10. Blauwblomme T, David O, Minotti L, Job AS, Chassagnon S, Hoffman D et al (2013) Prognostic value of insular lobe involvement in temporal lobe epilepsy: a stereoelectroencephalographic study. Epilepsia 54(9):1658-1667

11. Gras-Combe G, Minotti L, Hoffmann D, Krainik A, Kahane P, Chabardes S (2016) Surgery for nontumoral insular epilepsy explored by stereoelectroencephalography. Neurosurgery 79(4):578-588

12. Chiosa V, Granziera C, Spinelli L, Pollo C, Roulet-Perez E, Groppa S et al (2013) Successful surgical resection in nonlesional operculo-insular epilepsy without intracranial monitoring. Epileptic Disord 15(2):148-157

13. Ryvlin $P$ (2006) Avoid falling into the depths of the insular trap. Epileptic Disord 8(2):37-56

14. von Lehe M, Wellmer J, Urbach H, Schramm J, Elger CE, Clusmann H (2008) Insular lesionectomy for refractory epilepsy: management and outcome. Brain 132(4):1048-1056. https://doi. org/10.1093/brain/awp047

15. Nguyen DK, Nguyen DB, Malak R, Leroux JM, Carmant L, Saint-Hilaire JM et al (2009) Revisiting the role of the insula in refractory partial epilepsy. Epilepsia 50(3):510-520

16. Peltola ME, Trébuchon A, Lagarde S, Scavarda D, Carron R, Metsähonkala L et al (2020) Anatomoelectroclinical features of SEEG-confirmed pure insular-onset epilepsy. Epilepsy Behav $105: 15-18$

17. Wang X, Hu W, McGonigal A, Zhang C, Sang L, Zhao B et al (2019) Electroclinical features of insulo-opercular epilepsy: an SEEG and PET study. Ann Clin Transl Neurol 6(7):1165-1177

18. Ryvlin P, Kahane P (2005) The hidden causes of surgery-resistant temporal lobe epilepsy: extratemporal or temporal plus? Curr Opin Neurol 18(2):125-127

19. Wynford-Thomas R, Powell R (2016) Navigating the Island of Reil: how to understand the insular cortex. Pract Neurol. https:// doi.org/10.1136/practneurol-2016-001493

20. Fisher RS, Cross JH, French JA, Higurashi N, Hirsch E, Jansen FE et al (2017) Operational classification of seizure types by the international league against epilepsy: position paper of the ILAE commission for classification and terminology. Epilepsia 58(4):522-530

21. R Core Team (2018) R: A language and environment for statistical computing. R foundation for statistical computing, Vienna, Austria. Available online at https://www.R-project.org/

22. Zeng G (2017) On the existence of maximum likelihood estimates for weighted logistic regression. Commun Stat Theory Methods 46(22):11194-11203 
23. Kosmidis I (2014) Bias in parametric estimation: reduction and useful side-effects. Wiley Interdiscip Rev Comput Stat 6(3):185-196

24. William R, Dillon MG (1984) Multivariate analysis: methods and applications. Wiley, New York, p 608

25. Yushkevich PA, Piven J, Hazlett HC, Smith RG, Ho S, Gee JC et al (2006) User-guided 3D active contour segmentation of anatomical structures: significantly improved efficiency and reliability. Neuroimage 31(3):1116-1128

26. Rorden C, Fridriksson J, Karnath HO (2009) An evaluation of traditional and novel tools for lesion behavior mapping. Neuroimage 44(4):1355-1362. https://doi.org/10.1016/j.neuroimage. 2008.09.031

27. Manford M, Fish DR, Shorvon SD (1996) An analysis of clinical seizure patterns and their localizing value in frontal and temporal lobe epilepsies. Brain 119(1):17-40

28. Serles W, Pataraia E, Bacher J, Olbrich A, Aull S, Lehrner J et al (1998) Clinical seizure lateralization in mesial temporal lobe epilepsy: differences between patients with unitemporal and bitemporal interictal spikes. Neurology 50(3):742-747

29. Gallmetzer P, Leutmezer F, Serles W, Assem-Hilger E, Spatt J, Baumgartner C (2004) Postictal paresis in focal epilepsies-incidence, duration, and causes: a video-EEG monitoring study. Neurology 62(12):2160-2164

30. Foldvary-Schaefer N, Unnwongse K (2011) Localizing and lateralizing features of auras and seizures. Epilepsy Behav 20(2):160-166

31. Wang H, McGonigal A, Zhang K, Guo Q, Zhang B, Wang X et al (2020) Semiologic subgroups of insulo-opercular seizures based on connectional architecture atlas. Epilepsia 61:984

32. Singh R, Principe A, Tadel F, Hoffmann D, Chabardes S, Minotti L et al (2020) Mapping the insula with stereo-electroencephalography: the emergence of semiology in insula lobe seizures. Ann Neurol 88(3):477-488

33. Freri E, Matricardi S, Gozzo F, Cossu M, Granata T, Tassi L (2017) Perisylvian, including insular, childhood epilepsy: presurgical workup and surgical outcome. Epilepsia 58(8):1360-1369

34. Ryvlin P, Nguyen DK (2021) Insular seizures and epilepsies: ictal semiology and minimal invasive surgery. Curr Opin Neurol 34(2):153-165

35. Blenkmann A, Collavini S, Lubell J, Llorens A, Funderud I, Ivanovic J et al (2019) deviance detection in the human insula: an intracranial EEG study. Cortex 121:189-200

36. Trebaul L, Deman P, Tuyisenge V, Jedynak M, Hugues E, Rudrauf D et al (2018) Probabilistic functional tractography of the human cortex revisited. Neuroimage 181:414-429

37. Robinson CJ, Burton H (1980) Organization of somatosensory receptive fields in cortical areas $7 \mathrm{~b}$, retroinsula, postauditory and granular Insula of M. fascicularis. J Comp Neurol 192:69-92

38. Wang L, Mathews GC, Whetsell WO, Abou-Khalil B (2008) Hypermotor seizures in patients with temporal pole lesions. Epilepsy Res 82(1):93-98

39. Craig AD, Chen K, Bandy D, Reiman EM (2000) Thermosensory activation of insular cortex. Nat Neurosci 3(2):184-190

40. Pugnaghi M, Meletti S, Castana L, Francione S, Nobili L, Mai R et al (2011) Features of somatosensory manifestations induced by intracranial electrical stimulations of the human insula. Clin Neurophysiol 122(10):2049-2058

41. Mansournia MA, Geroldinger A, Greenland S, Heinze G (2018) Separation in logistic regression: causes, consequences, and control. Am J Epidemiol 187(4):864-870 